\title{
Does a deformation of special relativity imply energy dependent photon time delays?
}

\section{J.J. Relancio*, J.M. Carmona and J.L. Cortés}

Departamento de Física Teórica, Universidad de Zaragoza, Zaragoza 50009, Spain

E-mail: relancio@unizar.es, jcarmona@unizar.es, cortes@unizar.es

\begin{abstract}
Theoretical arguments in favor of energy dependent photon time delays from a modification of special relativity (SR) have met with recent gamma ray observations that put severe constraints on the scale of such deviations. We review the case of the generality of this theoretical prediction in the case of a deformation of SR and find that, at least in the simple model based on the analysis of photon worldlines which is commonly considered, there are many scenarios compatible with a relativity principle which do not contain a photon time delay. This will be the situation for any modified dispersion relation which reduces to $E=|\vec{p}|$ for photons, independently of the quantum structure of spacetime. This fact opens up the possibility of a phenomenologically consistent relativistic generalization of SR with a new mass scale many orders of magnitude below the Planck mass.
\end{abstract}

Corfu Summer Institute 2017 'School and Workshops on Elementary Particle Physics and Gravity'

2-28 September 2017

Corfu, Greece

${ }^{*}$ Speaker. 


\section{Introduction}

In the history of physics the exploration of smaller scales has required new theoretical frameworks. As a Russian doll, reality has revealed time after time shells of smaller scales. This leads us to the questions: Could we continue exploring closer the structure of Nature finding more and more shells? Is there a limit we cannot go beyond? If this limit exists, is it based on theoretical arguments or is it due to experimental reasons?

Any answer has to include not only the structure of matter. It has also to include the structure of the space-time, so it has to include gravity. One could wonder: if fundamental constituents of matter exist, does the same happen for space-time? Do space "atoms" exist? One has also to take into account that measuring smaller distances requires concentrating higher energies in small volumes, so one can not neglect the space-time curvature. In fact there is a limit in this amount of energy that we can concentrate due to the possible collapse into a black hole. So the gravitational interaction is indispensable.

One of the challenges for theoretical physics in our days is the unification of General Relativity (GR) and quantum theory. These two theories disagree in the role that space-time plays in them. In Quantum Field Theory (QFT), one assumes a given space-time and studies with detail the properties and the movement of particles in it. On the other hand, in GR, one assumes that the properties of matter and radiation are given and describes with detail the resultant space-time, that is, one studies the curvature. It is possible to have a quantum theory of gravitation considering an interaction mediated by a spin-2 particle (the graviton) that leads to the same equations that appear in GR. The main problem is that this theory is not renormalizable for energies comparable with the Planck energy.

One could wonder: if we have a theory for massive objects (GR) and another one for lighter particles (QFT), what do we need a Quantum Gravity Theory (QGT) for? In fact in order to describe the first instants of the universe one needs a theory that describes small particles with enormous energies. Also we do not know what happens inside a black hole. What happens with information once it crosses the event horizon? What happens when one comes to the singularity? To answer all this questions, we need a QGT.

There have been a lot of attempts to avoid the problems of inconsistency between GR and QFT: string theory, quantum loop gravity, supergravity, causal set theory... In most of these theories a minimum length appears, normally associated to the Planck length, and therefore there is an energy scale, the Planck one. The main problem is that there are no experimental observations that may help us to decide which is the correct approach to a fundamental theory of Quantum Gravity (QG). Deformed Special Relativity (DSR; for a review, see Ref. [1]) however, was developed as a low energy limit of a QGT that could have some experimental evidences. We can consider two ways to go beyond Special Relativity: a scheme in which there is a Lorentz invariance violation (LIV) and therefore a privileged reference frame, and a scheme in which there is still a relativity principle (RP). DSR is formulated in the last scheme. So the deformation of Deformed Special Relativity appears in a modified dispersion relation (MDR), modified Lorentz transformations (LT) and a modified, that is, non-additive, composition law for the momenta, but in such a way that the RP is satisfied. Our research is in these DSR theories.

In this theory, the Einstein relativity principle is generalized adding a new relativistic invariant 
to the speed of light $c$, the Planck length $l_{P}$. So this is why this theory is also called Doubly Special Relativity. The Planck length is normally considered as a minimum length.

There are numerous consequences of having a minimal length (see Ref. [2]). We can enumerate some of them:

- Space-time is quantized, discrete and non-continuous.

- Modified commutation rules appear since one can not measure with more precision than the Planck length and time.

- At these scales one can not determine the metric, so we lose the sense of curvature.

- All fundamental symmetries of SR and GR are only valid approximations for bigger scales than the Planck one.

- One can determine the number of dimensions of a space by taking all possible equidistant points minus 1. In $1 \mathrm{D}$ one has two points; in 2D, three points, and so on. So at the Planck scale we loose the naive sense of dimensions.

- The concept of point particle disappears. One can prove that since the size of particles is always bigger than the Planck length, their mass must be less than the Planck mass.

- Distinctions between real or virtual particles, matter-antimatter, matter-radiation... disappear at Planck scales.

At present it is impossible to see any of these previous implications. As we said before the main problem is the lack of observations and this can be understood by just comparing energies. If the Planck energy is $10^{19} \mathrm{GeV}$, the maximum particle accelerator energy is around $10^{4} \mathrm{GeV}$, and particles from cosmic rays come with an energy of $10^{11} \mathrm{GeV}$, how can we talk about phenomenology? The answer is that there could be amplifications at low energy, and over the past few years it has been realized that there are astrophysical observations that could be sensitive to such deviations Ref. [3].

The "foamy" structure of space-time could produce stochastic variations in the motion of particles. In many quantum gravity approaches, there are modified dispersion relations that can be written for energies much smaller than the Planck energy as

$$
m^{2}=C(p) \simeq E^{2}-\vec{p}^{2}-\xi_{n} E^{2}\left(\frac{E}{\Lambda}\right)^{n}
$$

where $n$ is an exponent giving the leading order behavior of the modification. With the Hamiltonian concept of speed $v=d E / d p$, this causes a time of flight delay given by

$$
\Delta t \sim\left(\frac{d}{c}\right) \xi_{n}\left(\frac{E}{\Lambda}\right)^{n}
$$

This delay can be measured for photons with different energies coming from a gamma ray burst. However, recent observations that consider possible time delays of photons impose strong restrictions of a first order $(n=1)$ deviation of SR (Refs. [4, 5, 6, 7, 8, 9, 10]). 
The previous experimental results suggest that there are no leading order planckian corrections in the dispersion relation of photons, at least if interpreted in the context of a Lorentz-violating scenario. However, the situation is again more subtle in the case of a deformation of SR. The discussion of energy-dependent photon time delays in DSR does not only involve the possible modification of the dispersion relation, but also the implementation of nontrivial translations between observers that are local to the emission and detection of the photons. Nontrivial translations are a necessary ingredient in a modification of SR that contains deformed Lorentz transformations in order to avoid inconsistencies with tests of locality, as the discussion in Ref. [11, 12] showed. This ingredient is quite natural in a theory which deforms the Poincare algebra of SR, and in fact it appears in the so-called relative locality framework [13], which is a proposal for the spacetime structure of DSR theories. In this proposal, it is the curvature of momentum space (which stems from the modified composition laws for momenta, unavoidable in DSR theories) that produce nonlocal effects in observers translated with respect to those which are local to an interaction.

Previous studies $[14,15,16]$ of photon time delays in the context of deformations of SR have used a model based on worldlines of free particles that propagate in a noncommutative spacetime, which is a simple way to implement nontrivial translations which are compatible with the deformed Poincaré algebra. They have obtained different conclusions about the existence of photon time delays, apparently depending on the type of noncommutative spacetime under analysis. In the present work we will consider the propagation in generic noncommutative spacetimes and establish the conditions for the absence of a photon time delay. As we will see, this may happen independently of the spacetime structure of the DSR theory, so that even first-order planckian corrections could be compatible with the above mentioned experimental results in a deformation of SR. As we will argue below, this fact opens up the possibility of a phenomenologically consistent deformation of SR with a new mass scale many orders of magnitude below the Planck mass.

The structure of the work is as follows: in Sec. 2 we will define the model and derive the expression for photon time delays. Then, in Sec. 3 we will see the main results obtained in Ref. [17] referring to two models very much studied in the literature, $\kappa$-Minkowski and Snyder spacetimes, checking that previous works (Refs. $[14,15,16])$ are particular cases of this model and give specific conditions for the absence of energy-dependent photon time delays. Finally, we will conclude in Sec. 4.

\section{A general model for photon time delays in noncommutative spacetimes}

The study of time delay effects in the propagation of particles in a modification of SR needs the specification of a spacetime. Effective field theories that violate Lorentz invariance [18, 19, 20,21] consider the classical, commutative, spacetime of SR. As argued above, this is no longer possible in the context of theories that modify SR but maintain a relativity principle, since they must incorporate nontrivial translations in spacetime. This is why noncommutativity is usually regarded as an appropriate feature of the spacetime formulation of DSR. While this ingredient is not yet worked out in full generality, ${ }^{1}$ there are specific examples of noncommutative spacetimes that have been explored and considered as benchmarks for the spacetime structure of a relativistic

\footnotetext{
${ }^{1}$ DSR theories are naturally formulated in momentum space [22, 23, 24].
} 
theory beyond SR. These include $\kappa$-Minkowski spacetime and the associated momentum space of the $\kappa$-Poincaré algebra, and also Snyder spacetime (both examples are reviewed in [25], and they were considered in relation with the calculation of photon time delays in Refs. [14, 15] and [16], respectively). In these cases, not only spacetime, but the whole phase space structure is modified with respect to the canonical phase space of SR.

If $(x, p)$ are the spacetime and momentum coordinates of a canonical phase space,

$$
\left\{p_{\mu}, x^{v}\right\}=\delta_{\mu}^{v}, \quad\left\{x^{\mu}, x^{v}\right\}=\left\{p_{\mu}, p_{v}\right\}=0,
$$

one can construct a nontrivial spacetime $(\tilde{x})$ by considering a linear combination of the $x$ coordinates with coefficients depending on the momentum variables $p$ and a new scale $M$, which must be necessarily introduced from dimensional arguments [26, 27]:

$$
\tilde{x}^{\mu}=x^{v} \varphi_{v}^{\mu}(p / M)
$$

One can refer to this new space as "noncommutative" in the sense that the Poisson bracket of two of these coordinates is not zero for a generic choice of coefficients $\varphi_{v}^{\mu}$. If we calculate the Poisson bracket of two spacetime coordinates we find

$$
\left\{\tilde{x}^{\mu}, \tilde{x}^{\nu}\right\}=-x^{\sigma} \varphi_{\rho}^{\mu} \frac{\partial \varphi_{\sigma}^{v}}{\partial p_{\rho}}+x^{\rho} \varphi_{\sigma}^{\nu} \frac{\partial \varphi_{\rho}^{\mu}}{\partial p_{\sigma}} .
$$

The worldlines of a particle $x^{\mu}(\tau)$, with $\tau$ an arbitrary parameter that flows along the worldline, can be obtained by applying the variational principle (as it is done in Ref. [17]), obtaining

$$
\tilde{x}^{\mu}(\tau)=\dot{\tilde{x}}^{\mu} \tau+\tilde{x}^{\mu}(0)=\varphi_{v}^{\mu}(p) \frac{\partial C}{\partial p_{v}} \tau+\tilde{x}^{\mu}(0) .
$$

We could also have obtained the previous result from the Poisson bracket of the function $C(p)$ defining the dispersion relation, $C(p)=m^{2}$, with $\tilde{x}^{\mu}$

$$
\dot{\tilde{x}}^{\mu}=\left\{C, \tilde{x}^{\mu}\right\}=\frac{\partial C}{\partial p_{v}}\left\{p_{v}, \varphi_{\rho}^{\mu}(p) x^{\rho}\right\}=\varphi_{v}^{\mu}(p) \frac{\partial C}{\partial p_{v}} .
$$

We can then define the velocity vector of the particle ${ }^{2}$

$$
\tilde{v}^{i}=\frac{\dot{\tilde{x}}^{i}}{\dot{\tilde{x}}^{0}}=\frac{\varphi_{v}^{i}\left(\partial C(p) / \partial p_{v}\right)}{\varphi_{v}^{0}\left(\partial C(p) / \partial p_{v}\right)}
$$

\subsection{Determination of time delays}

Our model considers the worldline of a free particle (a photon) that has its origin at a source (the emission point) and its end at a detector (the detection point). Since there is only one vector (the momentum $\vec{p}$ of the particle), the problem can be treated in $1+1$ dimensions without any loss of generality, so we will speak of its energy $E$ and its momentum $p \equiv|\vec{p}|{ }^{3}$ We assume that

\footnotetext{
${ }^{2}$ Note that the velocity is independent of the choice of the parameter $\tau$.

${ }^{3}$ Although we use the same notation $(p)$ for the four-momentum in $3+1$ and for the momentum in $1+1$, one can easily identify from the context which one is involved in the different equations.
} 
low-energy photons (in the limit $p / M \rightarrow 0$ ) behave as in SR: that is, their time of travel equals (in natural units, $c=1$ ) the distance between source and detector. In fact, we will define this distance $L$ from the emission at the source and absorption at the detector of low-energy photons, for which the functions $\varphi_{v}^{\mu} \rightarrow \delta_{v}^{\mu}$, so that they observe a commutative spacetime $\left(\tilde{x}^{\mu} \rightarrow x^{\mu}\right)$.

The time delay of a high-energy photon of momentum $p$ at the detector with respect to a lowenergy photon emitted "simultaneously at the same point" has two different sources: the modified dispersion relation $C(p)=0$, which defines the slope of the worldline, and the definition of simultaneity and spatial locality, which in a noncommutative spacetime, defined by the functions $\varphi(E, p)$ of Eq. (2.2), are relative concepts for observers whose spacetime origin do not coincide. In this case, there are two observers that must be brought into play: observer $A$, which is at the source, and observer $B$, which is at the detector. We define the origin of observer $A$ as the time and location of both a high and a low energy photon (this is the definition of a simultaneous emission at the source) and define the spacetime origin of observer $B$ to coincide with the detection of the low-energy photon.

One can obtain the translation relating the noncommutative spacetime coordinates of both observers from the trivial translations relating the commutative coordinates, $x^{B}=x^{A}-L, t^{B}=t^{A}-L$ and Eq. (2.2):

$$
\begin{aligned}
& \tilde{t}^{B}=\varphi_{0}^{0} t^{B}+\varphi_{1}^{0} x^{B}=\tilde{t}^{A}-L\left(\varphi_{0}^{0}+\varphi_{1}^{0}\right), \\
& \tilde{x}^{B}=\varphi_{0}^{1} t^{B}+\varphi_{1}^{1} x^{B}=\tilde{x}^{A}-L\left(\varphi_{0}^{1}+\varphi_{1}^{1}\right) .
\end{aligned}
$$

The worldline of the high energy particle for observer $A$ is

$$
\tilde{x}^{A}=\tilde{v} \tilde{t}^{A},
$$

since $\tilde{x}^{A}=0, \tilde{t}^{A}=0$ corresponds to the initial point of the worldline, and $\tilde{v}$ is obtained particularizing Eq. (2.6) to $1+1$ dimensions:

$$
\tilde{v}=\frac{\varphi_{0}^{1}(\partial C / \partial E)-\varphi_{1}^{1}(\partial C / \partial p)}{\varphi_{0}^{0}(\partial C / \partial E)-\varphi_{1}^{0}(\partial C / \partial p)}
$$

where the minus signs appear due to the fact that $p_{1}=-p^{1}=-p$, and so $\partial C / \partial p_{1}=-\partial C / \partial p$. We can now obtain the worldline for observer $B$ by applying Eqs. (2.7) and (2.8) to Eq. (2.9):

$$
\tilde{x}^{B}=\tilde{x}^{A}-L\left(\varphi_{0}^{1}+\varphi_{1}^{1}\right)=\tilde{v}\left[\tilde{t}^{B}+L\left(\varphi_{0}^{0}+\varphi_{1}^{0}\right)\right]-L\left(\varphi_{0}^{1}+\varphi_{1}^{1}\right) .
$$

The end of the worldline for observer $B$ happens at $\tilde{x}^{B}=0 .{ }^{4}$ We can then obtain the value of $\tilde{t}^{B}$ at that point from Eq. (2.11); this will give us the time-delay $\tilde{T} \equiv \tilde{t}^{B}\left(\tilde{x}^{B}=0\right)$ with respect to the arrival of the low-energy photon (that took place at $\tilde{t}^{B}=0$ ):

$$
\tilde{T}=\tilde{v}^{-1} L\left(\varphi_{0}^{1}+\varphi_{1}^{1}\right)-L\left(\varphi_{0}^{0}+\varphi_{1}^{0}\right)=L\left[\left(\varphi_{0}^{1}+\varphi_{1}^{1}\right) \frac{\varphi_{0}^{0}(\partial C / \partial E)-\varphi_{1}^{0}(\partial C / \partial p)}{\varphi_{0}^{1}(\partial C / \partial E)-\varphi_{1}^{1}(\partial C / \partial p)}-\left(\varphi_{0}^{0}+\varphi_{1}^{0}\right)\right] .
$$

Note that this equation is in fact valid for the time delay of any high-energy particle, independently of whether it is a photon or not. In the case of a particle of nonzero mass, Eq. (2.12) includes both the usual special relativistic time-delay of a massive particle and the time-delay induced by new physics with respect the arrival of a low-energy photon emitted simultaneously at the source.

\footnotetext{
${ }^{4} \mathrm{We}$ are assuming that the detector is at rest. Then the spatial location for the detection of the high energy particle and the low energy photon coincide.
} 


\subsection{Momenta as generators of translations in spacetime}

The functions $\varphi_{v}^{\mu}$ that were introduced in Eq. (2.2) are in correspondence with the Poisson brackets of energy and momentum with the noncommutative spacetime coordinates:

$$
\{E, \tilde{t}\}=\left\{E, \varphi_{v}^{0} x^{v}\right\}=\varphi_{0}^{0}, \quad\{E, \tilde{x}\}=\varphi_{0}^{1}, \quad\{p, \tilde{t}\}=-\varphi_{1}^{0}, \quad\{p, \tilde{x}\}=-\varphi_{1}^{1},
$$

where again the minus signs appear due to $p_{1}=-p^{1}=-p$.

The transformations Eqs. (2.7) and (2.8) between observers $A$ and $B$ can then be written in the form

$$
\begin{aligned}
& \tilde{t}^{B}=\tilde{t}^{A}-L\{E, \tilde{t}\}+L\{p, \tilde{t}\}, \\
& \tilde{x}^{B}=\tilde{x}^{A}-L\{E, \tilde{x}\}+L\{p, \tilde{x}\} .
\end{aligned}
$$

These transformations correspond, then, to translations in the noncommutative spacetime which are generated by the energy and the momentum, even if the $\left(\tilde{x}^{\mu}, p_{v}\right)$ phase space is noncanonical. This was in fact the approach taken in the analyses of Refs. $[14,15]$ and [16].

The formula for the time-delay Eq. (2.12) can then be written in terms of Poisson brackets in the following form:

$$
\tilde{T}=(L\{E, \tilde{x}\}-L\{p, \tilde{x}\}) \cdot\left(\frac{(\partial C / \partial E)\{E, \tilde{t}\}+(\partial C / \partial p)\{p, \tilde{t}\}}{(\partial C / \partial E)\{E, \tilde{x}\}+(\partial C / \partial p)\{p, \tilde{x}\}}\right)-L\{E, \tilde{t}\}+L\{p, \tilde{t}\} .
$$

In a commutative spacetime, we have $\{E, t\}=1,\{E, x\}=0,\{p, t\}=0,\{p, x\}=-1$, and then Eq. (2.16) gives

$$
T=-L\left(1+\frac{\partial C / \partial E}{\partial C / \partial p}\right)
$$

This expression contains of course the particular case of SR, $T=-L(1-E / p)$, which is zero for photons.

\section{Results}

In Ref. [17] two models of noncommutativity are studied, one where the modification starts at first order in the high energy scale whilst the second one starts at second order. The first one is $\kappa$-Minkowski spacetime, which has been very much studied in relation with DSR theories. The nontrivial Poisson bracket of spacetime coordinates is

$$
\left\{\tilde{x}^{0}, \tilde{x}^{i}\right\}=-\frac{\tilde{x}^{i}}{\Lambda}
$$

The other model we have studied is Snyder spacetime, which is a covariant model. The Poisson bracket of two spacetime coordinates is the Lorentz generator

$$
\left\{\tilde{x}^{\mu}, \tilde{x}^{v}\right\}=\frac{J^{\mu v}}{\Lambda^{2}} .
$$

It was proposed by Snyder in 1947 (Ref. [28]) as a way to avoid the divergences in QFT. However, since renormalization solved this problem, this algebra was forgotten until noncommutative spacetimes were studied as an attempt of a QGT. Due to covariance, in all choices of $\varphi$ the dispersion relation (DR) and LT are those of SR. 
In Ref. [17] three different basis of $\kappa$-Minkowski (bicrossproduct, Magueijo-Smolin and classical basis) and the representation of Snyder and Maggiore of the Snyder spacetime were studied. In all of those cases it was seen that the time delay is proportional to

$$
L[(1+(\partial C / \partial E) /(\partial C / \partial p)]
$$

i.e., to $(L / v-L)$, where $v$ is the velocity of propagation of the high energy particle in the commutative spacetime,

$$
v=-\frac{\partial C / \partial p}{\partial C / \partial E} .
$$

This result can in fact be obtained from the general expression (2.12):

$$
\tilde{T}=L\left[\left(\varphi_{0}^{1}+\varphi_{1}^{1}\right) \frac{\varphi_{0}^{0}+\varphi_{1}^{0} v}{\varphi_{0}^{1}+\varphi_{1}^{1} v}-\left(\varphi_{0}^{0}+\varphi_{1}^{0}\right)\right]=\frac{L\left(\varphi_{0}^{0} \varphi_{1}^{1}-\varphi_{0}^{1} \varphi_{1}^{0}\right)}{\varphi_{0}^{1}+\varphi_{1}^{1} v}(1-v)=\frac{\varphi_{0}^{0} \varphi_{1}^{1}-\varphi_{0}^{1} \varphi_{1}^{0}}{\varphi_{1}^{1}+\varphi_{0}^{1} / v} L\left(\frac{1}{v}-1\right)
$$

Then we see that the time delay is just the naive result taking into account the (possible) energy dependence of the velocity of propagation, multiplied by a factor which involves the dependence on the details of the noncommutative spacetime.

In the case of photons (for which there is no time delay in SR), and in an expansion in powers of $(1 / M)$, the time delay will be proportional to $(1 / M)$ [or $\left(1 / M^{2}\right)$ if corrections start at second order]. But the factor given by expression (3.3) is already of order $(1 / M)$ [or $\left(1 / M^{2}\right)$ for a second order correction]. Then the dominant contribution to the time delay will be independent of the details of the noncommutative spacetime and will be determined just by the modification of the dispersion relation $C(p)$. This is the reason why the dominant contribution to photon time delays are the terms in the modified dispersion relation, which is compatible with the results obtained in Refs. [14, 15, 16].

The condition for the absence of time delays is, therefore, $v=1$. But, taking differentials in the photon dispersion relation, $C(E, p)=0$, one gets

$$
-\left.\frac{\partial C / \partial p}{\partial C / \partial E}\right|_{C(E, p)=0}=\frac{d E(p)}{d p}
$$

where $E(p)$ is the solution of $C(E, p)=0$. Then the absence of time delay requires that for photons, $E(p)=p$. The possibility to have modified dispersion relations that reduce to $E(p)=p$ for photons was already noted in Ref. [29]. Quite generally, if one considers an expansion in powers of $(1 / M)$

$$
C(E, p)=E^{2}-p^{2}+\sum_{n} \frac{1}{M^{n}} C^{(n)}(E, p),
$$

then the conditions that the dispersion relation has to satisfy so that photons do not show a time delay are

$$
\left.C^{(n)}(E, p)\right|_{p=E}=0
$$

This is consistent with the result obtained by a general calculation of time delays for a first (or a second) order correction to SR showed in Ref. [17].

In the case of a massive high energy particle one would have a time delay in SR due to the energy dependence of the velocity of propagation of a massive particle that could be modified due 
to corrections to SR. Even if the dispersion relation were not modified (and, therefore, $v$ were the same as in SR), one would have a time delay for massive particles which would be the mass dependent time delay of SR, multiplied by a factor depending on the details of the noncommutativity proportional to the ratio $E / M$ [or $\left(E^{2} / M^{2}\right)$ ]. In order to have an observation sensitive to the noncommutativity one would require a measurement of the mass dependent time delay with a precision of order $(E / M)$ [or $\left.\left(E^{2} / M^{2}\right)\right]$.

\section{Conclusions}

The main result obtained in this work is that it is possible to go beyond SR without observable time delays, even for a deformation of SR (that is, in the presence of a relativity principle). We have identified that this is the case whenever the velocity of propagation of photons in the commutative spacetime is independent of the energy, which would be the naive conclusion forgetting about the effects of a nontrivial spacetime. Considering a model based on worldlines of particles in a noncommutative spacetime we have found several examples with no time delay for photons.

Although the standard studies of Lorentz violation assume a commutative spacetime, one could consider a noncommutative spacetime also in this case. The difference between a scenario with LIV and a relativistic theory is that in the latter case one has to keep a (deformed) Poincare symmetry, which requires a consistency of the modified dispersion relation and the nontrivial implementation of translational symmetry which fixes the noncommutativity of spacetime. On the contrary, in the case of LIV one can choose independently the dispersion relation and the noncommutativity of spacetime. The absence of time delays is a property which depends exclusively on the dispersion relation and then applies indistinctly to both cases.

All the results of this work are based on a noncommutative spacetime as the appropriate model to calculate time delays induced by departures from SR. This is the most common framework that has been considered in previous analyses $[14,15,16]$ in order to implement nontrivial translations in the context of deformations of SR, and it is the simplest way to introduce a relativity of locality for the emission and detection processes, which are local only for the observers at the source and at the detector, respectively. However, the framework introduced in Ref. [13] considers a relativity of the locality associated to the interaction of particles as an alternative to a formulation based on spacetime noncommutativity. In this case one should consider the nonlocality of the interactions responsible for the emission and detection of particles as the appropriate model to study the possibility to have observable time delays.

One could take the main outcome of this work as bad news from a phenomenological point of view since it means that if the possible departures from SR induced by quantum gravity are such that there is no time delay for photons the only phenomenological window to deformations of SR (if they do not include the possibility of photon birefringence) gets closed. Alternatively one can take the result as good news since it means that the strong constraints on the mass scale parametrizing the departures from SR obtained from the absence of observations of time delays for photons are not applicable with full generality. Such constraints would not necessarily imply that quantum gravity corrections start at least at second order of the Planck mass (which is still a possibility), but would be compatible with first-order modifications of SR which do not produce photon time delays. 
Moreover, since all the constraints based on the use of effective field theory to go beyond SR $[18,19,20,21]$ apply only to an scenario without the presence of a relativity principle, the result obtained in this paper opens up the interesting possibility of a scale of deformation of special relativity much smaller than its simplest estimate (the Planck mass) without any phenomenological inconsistency. The simplest estimate is based on naturalness but we already know that this argument fails in the case of the vacuum energy, and we have also hints that it also fails in the estimate for the mass of elementary scalars (the standard model (SM) Higgs particle seems to be much lighter than the scale limiting the domain of validity of the SM). Then it seems reasonable to explore the possibility of some mechanism generating a scale for the departures from SR much smaller than this simplest estimate. If this were the case then one would have to reconsider searches of possible signals of quantum gravity that may have been discarded based on the assumption that the scale for such signals is of the order of the Planck mass.

\section{Acknowledgments}

This work is supported by the Spanish MINECO FPA2015-65745-P (MINECO/FEDER) and Spanish DGIID-DGA Grant No. 2015-E24/2. We acknowledge useful conversations with Niccolò Loret.

\section{References}

[1] G. Amelino-Camelia, Quantum-Spacetime Phenomenology, Living Rev.Rel. 16 (2013) 5 [0806.0339].

[2] C. Schiller, Does matter differ from vacuum?, gr-qc/9610066.

[3] D. Mattingly, Modern tests of Lorentz invariance, Living Rev.Rel. 8 (2005) 5 [gr-qc/ 0502097 ].

[4] MAGic, Other Contributors collaboration, J. Albert et al., Probing Quantum Gravity using Photons from a flare of the active galactic nucleus Markarian 501 Observed by the MAGIC telescope, Phys. Lett. B668 (2008) 253 [0708.2889].

[5] M. Martinez and M. Errando, A new approach to study energy-dependent arrival delays on photons from astrophysical sources, Astropart. Phys. 31 (2009) 226 [0803.2120].

[6] Fermi GBM/LAT collaboration, A. A. Abdo et al., A limit on the variation of the speed of light arising from quantum gravity effects, Nature 462 (2009) 331 [0 908 . 1832].

[7] HESS collaboration, A. Abramowski et al., Search for Lorentz Invariance breaking with a likelihood fit of the PKS 2155-304 Flare Data Taken on MJD 53944, Astropart. Phys. 34 (2011) 738 [1101.3650].

[8] R. J. Nemiroff, R. Connolly, J. Holmes and A. B. Kostinski, Bounds on Spectral Dispersion from Fermi-detected Gamma Ray Bursts, Phys. Rev. Lett. 108 (2012) 231103 [1109. 5191].

[9] V. Vasileiou, A. Jacholkowska, F. Piron, J. Bolmont, C. Couturier, J. Granot et al., Constraints on Lorentz Invariance Violation from Fermi-Large Area Telescope Observations of Gamma-Ray Bursts, Phys. Rev. D87 (2013) 122001 [1305.3463].

[10] V. Vasileiou, J. Granot, T. Piran and G. Amelino-Camelia, A Planck-scale limit on spacetime fuzziness and stochastic Lorentz invariance violation, Nature Phys. 11 (2015) 344. 
[11] G. Amelino-Camelia, M. Matassa, F. Mercati and G. Rosati, Taming Nonlocality in Theories with Planck-Scale Deformed Lorentz Symmetry, Phys. Rev. Lett. 106 (2011) 071301 [1 006.2126 ].

[12] S. Hossenfelder, Bounds on an energy-dependent and observer-independent speed of light from violations of locality, Phys. Rev. Lett. 104 (2010) 140402 [1004.0418].

[13] G. Amelino-Camelia, L. Freidel, J. Kowalski-Glikman and L. Smolin, The principle of relative locality, Phys.Rev. D84 (2011) 084010 [1101.0931].

[14] G. Amelino-Camelia, N. Loret and G. Rosati, Speed of particles and a relativity of locality in K-Minkowski quantum spacetime, Phys. Lett. B700 (2011) 150 [1102.4637].

[15] N. Loret, Exploring special relative locality with de Sitter momentum-space, Phys. Rev. D90 (2014) 124013 [1404.5093].

[16] S. Mignemi and A. Samsarov, Relative-locality effects in Snyder spacetime, Phys. Lett. A381 (2017) 1655 [1610.09692].

[17] J. M. Carmona, J. L. Cortes and J. J. Relancio, Does a deformation of special relativity imply energy dependent photon time delays?, Class. Quant. Grav. 35 (2018) 025014 [1702 . 03669 ].

[18] V. A. Kostelecky and N. Russell, Data Tables for Lorentz and CPT Violation, Rev. Mod. Phys. 83 (2011) 11 [0801.0287].

[19] J. C. Long and V. A. Kostelecky, Search for Lorentz violation in short-range gravity, Phys. Rev. D91 (2015) 092003 [1412.8362].

[20] A. Kostelecky, E. Lunghi and A. R. Vieira, Lorentz violation and deep inelastic scattering, 1610.08755 .

[21] V. A. Kostelecky, A. C. Melissinos and M. Mewes, Searching for photon-sector Lorentz violation using gravitational-wave detectors, Phys. Lett. B761 (2016) 1 [1608 . 02592].

[22] G. Amelino-Camelia, Doubly special relativity: First results and key open problems, Int.J.Mod.Phys. D11 (2002) 1643 [gr-qc/ 0210063$].$

[23] J. Magueijo and L. Smolin, Lorentz invariance with an invariant energy scale, Phys. Rev. Lett. 88 (2002) 190403 [hep-th/0112090].

[24] G. Amelino-Camelia, Kinematical solution of the UHE - cosmic - ray puzzle without a preferred class of inertial observers, Int. J. Mod. Phys. D12 (2003) 1211 [astro-ph/0209232].

[25] J. Kowalski-Glikman and S. Nowak, Noncommutative space-time of doubly special relativity theories, Int. J. Mod. Phys. D12 (2003) 299 [hep-th/ 020424 5].

[26] S. Meljanac, D. Meljanac, F. Mercati and D. Pikutï̈Ğ, Noncommutative spaces and PoincarÃl' symmetry, Phys. Lett. B766 (2017) 181 [1610.06716].

[27] N. Loret, S. Meljanac, F. Mercati and D. Pikutï̈Ğ, Vectorlike deformations of relativistic quantum phase-space and relativistic kinematics, Int. J. Mod. Phys. D26 (2017) 1750123 [1610.08310].

[28] H. S. Snyder, Quantized space-time, Phys. Rev. 71 (1947) 38.

[29] S. Hossenfelder, A note on theories with a minimal length, Class. Quant. Grav. 23 (2006) 1815 [hep-th/0510245]. 